\title{
THE MINIMAL HARMONIC FUNCTIONS OF SOJOURN PROCESSES OF CERTAIN FINITE STATE MARKOV CHAINS
}

\author{
E. BOLTHAUSEN
}

\begin{abstract}
To a finite state, discrete time Markov chain a new chain is constructed which counts the number of sojourns in each state. This sojourn process is a transient Markov chain. The minimal harmonic functions of this chain are identified.
\end{abstract}

1. Introduction. Let $I$ be a finite set of $s$ states and $Z_{n}, n \in \mathrm{N}$ be a sequence of independent identically distributed $I$-valued random variables and let $p_{i}=\operatorname{Pr}\left(Z_{n}=i\right)$. To $Z_{n}$ we associate a sequence of random variables $X_{n}=$ $\left(\chi_{i}^{(n)}, \ldots, \chi_{s}^{(n)}\right)$ with values in $\mathbf{N}_{0}^{s}\left(\mathbf{N}_{0}\right.$ the set of nonnegative integers) by

$$
\chi_{i}^{(n)}=\sum_{m=1}^{n} 1_{i}\left(Z_{m}\right)
$$

Clearly, $X_{n}$ is a transient Markov chain. D. Blackwell and D. Kendall calculated in [1] the Martin boundary of this chain. (See also [5, p. 394].) (Actually, Blackwell and Kendall calculated the Martin boundary of Pólya's urn scheme, but it turns out that Pólya's urn scheme is a $h$-path-transformation of $X_{n}$.) The boundary can be identified with the simplex

$$
\Delta_{s}=\left\{t=\left(t_{1}, \ldots, t_{s}\right): 0 \leqslant t_{i}, \sum_{i=1}^{s} t_{i}=1\right\} .
$$

The minimal nonnegative harmonic function corresponding to $t \in \Delta_{s}$ is given by

$$
\mathbf{N}_{0}^{s} \ni\left(x_{1}, \ldots, x_{s}\right) \rightarrow \prod_{i=1}^{s}\left(t_{i} / p_{i}\right)^{x_{i}} .
$$

It is the purpose of this paper to generalize this result to the case where $Z_{n}$ is a finite state Markov chain with all transition probabilities nonvanishing. To such a chain we associate $X_{n}$ as above, but one has to take the joint variables $Y_{n}=\left(X_{n}, Z_{n}\right)$ to get a Markov chain. We call $Y_{n}$ the sojourn process of $Z_{n}$. The minimal nonnegative harmonic functions again correspond bijective to points in $\Delta_{s}$, but the connection is more complicated.

Received by the editors May 9, 1978 and, in revised form, November 27, 1978. AMS (MOS) subject classifications (1970). Primary 60J05, 60J45; Secondary 60J50.

Key words and phrases. Markov chain, sojourn times, harmonic functions, Martin boundary. 
2. Notations and statements. Let $I=\{1, \ldots, s\}$ be as above and $P=$ $\left(p_{i j}\right)_{i, j \in I}$ be a matrix of transition probabilities on $I$ which all are nonvanishing. Let $\delta_{i} \in \mathbf{N}_{0}^{s}$ have components 0 except the $j$ th which is 1 . To $P$ we associate a transition probability matrix $Q$ on $\tilde{I}=\mathbf{N}_{0}^{s} \times I$ : if $x, y \in \mathbf{N}_{0}^{s}$ then

$$
q_{(x, i),(y, j)}= \begin{cases}p_{i j} & \text { if } y=x+\delta_{j}, \\ 0 & \text { else. }\end{cases}
$$

We call a function $h: I \rightarrow \mathbf{R}^{+}$harmonic if for all $(x, i) \in I$,

$$
h(x, i)=\sum_{j \in I} p_{i j} h\left(x+\delta_{j}, j\right)=\sum_{(y, i) \in \tilde{I}} q_{(x, i),(y, j)} h(y, j) .
$$

(We shall only consider nonnegative harmonic functions.) Let $\mathcal{H}$ denote the set of harmonic functions which are not identically 0 . A harmonic function $h$ is called minimal if whenever $h^{\prime} \in \mathcal{H}$ and $h^{\prime} \leqslant h$ (i.e., $h^{\prime}(x, i) \leqslant h(x, i)$ for all $(x, i) \in \tilde{I})$ then there is a constant $c>0$ with $h^{\prime}=c h$. Let $\mathcal{K}_{e}$ be the set of minimal harmonic functions.

We shall give a complete description of the elements in $\mathcal{K}_{e}$.

If $u=\left(u_{1}, \ldots, u_{s}\right) \in\left(\mathbf{R}^{+}\right)^{s}-\{0\}$ let $v=\left(v_{1}, \ldots, v_{s}\right)$ be defined by $v_{i}=$ $\Sigma_{j} p_{i j} u_{j} . u$ and $v$ will always be connected in this way. Clearly, from the assumption that $p_{i j}>0$ for all $i, j \in I$, we have $v_{i}>0$ for all $i$. To $u \in\left(\mathbf{R}^{+}\right)^{s}$ - $\{0\}$ we associate a function $h_{u}: \tilde{I} \rightarrow \mathbf{R}^{+}$by $h_{u}(x, i)=v_{i} \Pi_{j=1}^{s}\left(u_{j} / v_{j}\right)^{x_{j}}$.

Theorem 1. $\mathcal{H}_{e}=\left\{h_{u}: u \in\left(\mathbf{R}^{+}\right)^{s}-\{0\}\right\}$.

The proof is deferred to $\$ 3$.

We consider now the subsets $\mathscr{D}=\{h \in \mathcal{H}: h(0,1)=1\}$ and $\mathscr{D}_{e}=\mathscr{D} \cap$ $\mathcal{H}_{e}$. It is easy to see that for $h \in \mathcal{K}, h(0,1)>0$. (In fact, if $h(0,1)=0$ then from $h(0,1)=\sum p_{1 j} h\left(\delta_{j}, j\right)$ one obtains $h\left(\delta_{j}, j\right)=0$ for all $j$ and then $h(0, j)=$ 0 for all $j$ and a straightforward conclusion gives $h=0$.) So each $h \in \mathcal{H}$ has a unique representation $h=c h^{\prime}$ where $c>0$ and $h^{\prime} \in \mathscr{D}$.

If $\mathcal{H}$ is equipped with the topology of pointwise convergence, $\mathscr{D}$ is a compact convex subset of $\mathbf{R}^{\tilde{I}}$ with $\mathscr{D}_{e}$ as the set of extremal points. Now $h_{u} \in \mathscr{D}$ if and only if $\Sigma_{j=1}^{s} p_{1 j} u_{j}=1$. Let $\tilde{\Delta}_{s}=\left\{u \in\left(\mathbf{R}^{+}\right)^{s}: \Sigma p_{1 j} u_{j}=1\right\}$, then the topology on $\mathscr{D}_{e}$ corresponds to the usual topology on $\left(\mathbf{R}^{+}\right)^{s}$ under the correspondence $\tilde{\Delta}_{s} \ni u \mapsto h_{u} \in \mathscr{D}_{e}$. From general Choquet representation theory one thus obtains (see e.g. [2]):

THEOREM 2. To any $h \in \mathscr{D}$ there exists a unique probability measure $\mu$ on the Borel sets of $\tilde{\Delta}_{s}$ such that

$$
h(x, i)=\int h_{u}(x, i) \mu(d u)
$$

for all $(x, i) \in I$.

One would like to have a probabilistic interpretation of the elements in $\tilde{\Delta}_{s}$. Let us first fix some notations: Let $\Omega=I^{N_{0}}$ equipped with the product $\sigma$-algebra which we denote by $\mathfrak{A}$. For $i \in I$ let $P^{i}$ be the law on $(\Omega, \mathfrak{A})$ which 
governs the Markov chain with transition probabilities $p_{i j}$ and starting point $i \in I$. Let $Z_{n}, n \in \mathbf{N}_{0}$, be the projections of $\Omega$ on its factors and let $X_{n}$ be defined as in the introduction $\left(X_{0}=0\right)$. Then $Y_{n}=\left(X_{n}, Z_{n}\right)$ is a transient Markov chain with state space $\tilde{I}$ and transition probabilities $q_{\ldots, .}$. We call $Y_{n}$, $n \in \mathrm{N}_{0}$ the sojourn process. Actually we can start $Y_{n}$ in any point $(x, i) \in \tilde{I}$ simply by replacing $X_{n}$ by $x+X_{n}$ and starting $Z_{n}$ in $i$. We denote by $Q^{(x, i)}$ the thus induced law on $\tilde{I}^{\mathbf{N}_{0}}$. To $u \in \tilde{\Delta}_{s}$ we associate Markov chain transition probabilities $p_{i j}^{u}=p_{i j} u_{j} / v_{i}$. Let $\pi^{u}=\left(\pi_{1}^{u}, \ldots, \pi_{s}^{u}\right)$ be the stationary distribution of the $p_{i j}^{u}$, that is $\pi^{u}$ is the unique element in $\Delta_{s}\left(\Delta_{s}\right.$ defined as in the introduction) with $\pi_{j}^{u}=\sum_{i=1}^{s} \pi_{i}^{u} p_{i j}^{u}$. One thus obtains a mapping $\Psi: \tilde{\Delta}_{s} \rightarrow \Delta_{s}$ defined by $\Psi(u)=\pi^{u}$.

\section{Proposition 3. $\Psi$ is a homeomorphism.}

The proof is deferred to $\S 3$.

It is now easy to see that the $h$-path transformation (see, e.g., [5]) of the $Y_{n}$ process by $h_{u}$ is exactly the sojourn process associated with the transition probabilities $p_{i j}^{u}$. So to any probability measure $\mu$ on $\Delta_{s}$ there corresponds exactly one element in $\mathscr{D}_{e}$, namely $h_{\Psi^{-1}(\mu)}$, such that the $h$-path transformation of the $Y_{n}$ process with this element is the sojourn process of a chain with stationary distribution $\mu$.

The present work has relations to the investigations of harmonic functions and Martin boundaries of the so called space-time process $\left(Z_{n}, n\right)$, where $Z_{n}$, $n \in \mathbf{N}_{\mathbf{0}}$ are recurrent Markov chains (much more general than ours, see [6]). Indeed, $n$ is simply $\sum_{i=1}^{s} \chi_{i}^{(n)}$ in our notation. So our sojourn processes obviously carry more information than the space-time processes.

There is also a connection with certain conditionings investigated by Darroch and Seneta [3]. Let $I_{0}$ be a proper nonvoid subset of $I$ and let $T=\inf \left\{n \in \mathbf{N}: Z_{n} \notin I_{0}\right\}$. Then it is easy to see that $Z_{0}, \ldots, Z_{n}$ conditioned on $\{T>n\}$ is a nonhomogeneous Markov chain. Darroch and Seneta have shown that for $n \rightarrow \infty$ the transition probabilities of this chain converge to stationary transition probabilities $q_{i j}$ on $I_{0}$, that is, for $l \in I_{0}$ and each $k \in \mathbf{N}_{0}$,

$$
\lim _{n \rightarrow \infty} P^{l}\left(X_{k+1}=j \mid X_{k}=i, T>n\right)=q_{i j}
$$

One can show that $q_{i j}=p_{i j}^{u}$ where $u=\Psi^{-1}(\mu)$ where $\mu$ is a probability on $I_{0}$ such that the Donsker-Varadhan information $I(\mu)$ (see [4]) is minimal among probability measures which are carried by $I_{0}$.

Now $T>n$ is simply a condition on the sojourn times of $Z_{n}: \Sigma_{i \notin I_{0}} \chi_{i}^{(n)}=$ 0 . One may consider other conditionings: Let $\Delta_{0}$ be a closed subset of $\Delta_{s}$ and $l \in I$ such that $Q^{(0, l)}\left(X_{n} / n \in \Delta_{0}\right)>0$ for sufficiently large $n$. Then one can take elementary conditionings on $\left\{X_{n} / n \in \Delta_{0}\right\}$. In general, $Z_{0}, \ldots, Z_{n}$ is not a Markov chain, but $Y_{0}, \ldots, Y_{n}$ is. One may conjecture that, as in the Darroch-Seneta case, if $I(\mu)$ attains a minimum on $\Delta_{0}$ which is uniquely 
attained in $\mu_{0}$, then

$$
\lim _{n \rightarrow \infty} Q^{(0, l)}\left(Y_{k+1}=(y, j) \mid Y_{k}=(x, i), X_{n} / n \in \Delta_{0}\right)=q_{(x, i),(y, j)}^{u}
$$

where $q_{(x, i),(y, j)}^{u}$ are constructed as above from the $p_{i j}^{u}$ and where $u=\Psi^{-1}\left(\mu_{0}\right)$. As far as I know, such conditionings have not been considered in the literature as yet.

3. Proofs. For $x \in \mathbf{N}_{0}^{s}$ we associate a map $\phi_{x}: \mathcal{H} \rightarrow \mathcal{H}$ by $\left(\phi_{x} h\right)(y, i)=h(x$ $+y, i)$. It is obvious that $\phi_{x} h \in \mathcal{H}$ if $h \in \mathcal{K}$. We simply write $\phi_{i}$ for $\phi_{\delta_{i}}$. For $i \in I$ let $\tau_{i}:(\tilde{I})^{\mathbf{N}_{0}} \rightarrow \overline{\mathbf{N}}_{\mathbf{0}}=\mathbf{N}_{0} \cup\{\infty\}$ be defined by

$$
\tau_{i}\left(\left(x_{0}, i_{0}\right),\left(x_{1}, i_{1}\right), \ldots\right)=\inf \left\{n \in \mathbf{N}_{0}: x_{n, i} \geqslant 1\right\}
$$

where $x_{n, i}$ is the $i$ th component of $x_{n}$. Clearly $\tau_{i}$ is a stopping time for the $Y_{n}$ process and $Q^{(x, j)}\left(\tau_{i}<\infty\right)=1$ for all $(x, j) \in \tilde{I}$ and $Q^{(x, j)}\left(\tau_{i}=0\right)=1$ if $x_{i} \geqslant 1$. For $h \in \mathcal{H}$ and $i \in I$ let $\Lambda_{i} h$ be the function $\tilde{I} \rightarrow \mathbf{R}^{+}$defined by

$$
\left(\Lambda_{i} h\right)(x, j)=E^{(x, j)}\left(h\left(Y_{\tau_{i}}-\delta_{i}\right)\right)
$$

where $(y, l)-\delta_{i}=\left(y-\delta_{i}, l\right)$.

Lemma 1. If $h \in \mathcal{H}$ then $\Lambda_{i} h \in \mathcal{H}$.

Proof. Let $(x, l) \in \tilde{I}$,

$$
\begin{aligned}
\sum_{(y, j) \in \tilde{I}} q_{(x, l)(y, j)}\left(\Lambda_{i} h\right)(y, j) & \\
= & \sum_{j \in I} p_{l j} E^{\left(x+\delta_{j} j\right)} h\left(Y_{\tau_{i}}-\delta_{i}\right)=E^{(x, l)} h\left(Y_{\hat{\tau}_{i}}-\delta_{i}\right)
\end{aligned}
$$

where $\hat{\tau}_{i}=\max \left(\tau_{i}, 1\right)$. Now $x_{i}=0$ if and only if $\tau_{i} \geqslant 1 Q^{(x, l)}$ a.s. So if $x_{i}=0$ the above expression becomes

$$
E^{(x, l)} h\left(Y_{\tau_{1}}-\delta_{i}\right)=\left(\Lambda_{i} h\right)(x, l)
$$

and if $x_{i} \geqslant 1$ it equals $E^{(x, l)} h\left(Y_{1}-\delta_{i}\right)$ which by harmonicity is

$$
h\left(\left(x-\delta_{i}, l\right)\right)=E^{(x, l)} h\left(Y_{0}-\delta_{i}\right)=E^{(x, l)}\left(Y_{\tau_{i}}-\delta_{i}\right)=\left(\Lambda_{i} h\right)(x, l) .
$$

So the lemma is proved.

Clearly, one has for $h \in \mathcal{H}$,

$$
\left(\Lambda_{i} \phi_{i} h\right)(x, j)=E^{(x, j)} h\left(Y_{\tau_{i}}\right)<h(x, j)
$$

the last inequality because $h\left(Y_{n}\right), n \in \mathrm{N}_{0}$ is a nonnegative martingale and $\tau_{i}$ is a stopping time.

$$
\phi_{i} \Lambda_{i} h=h \text {. }
$$

LEMMA 2. If $h \in \mathcal{H}_{e}$ then $\phi_{i} h=0$ or $\phi_{i} h \in \mathcal{H}_{e}$.

Proof. From (3.1), $\Lambda_{i} \phi_{i} h \leqslant h$ so $\Lambda_{i} \phi_{i} h=c h$ for some $c \in[0,1]$. If $c<1$ then it follows from the fact that $\left(\Lambda_{i} \phi_{i} h\right)(x, j)=h(x, j)$ for all $(x, j)$ with $x_{i}>1$ that $h(x, j)=0$ for $x_{i} \geqslant 1$, so $\Lambda_{i} \phi_{i} h=0$ and therefore from (3.2), $\phi_{i} h=0$. If $c=1$, let $h^{\prime} \in \mathcal{H}, h^{\prime} \leqslant \phi_{i} h$. It follows that $h=\Lambda_{i} \phi_{i} h>\Lambda_{i} h^{\prime}$ so 
there is a $c^{\prime} \in[0,1]$ with $\Lambda_{i} h^{\prime}=c h$ and so $h^{\prime}=\phi_{i} \Lambda_{i} h^{\prime}=c^{\prime} \phi_{i} h$, and it is proved that $\phi_{i} h$ is extremal.

LemMA 3. Let $h \in \mathcal{H}_{e}$ and $x, y \in \mathbf{N}_{0}^{s}$ with $\Sigma_{i \in I} x_{i} y_{i}=0$ and $\phi_{x} h \neq 0$ and $\phi_{y} h \neq 0$. Then $\phi_{x+y} h \neq 0$.

Proof. We proceed by induction on $|x|=\sum_{i \in I} x_{i} \cdot|x|=0$ is clear. If $i$ is such that $y_{i}=0$ and $\sum x_{i} y_{i}=0$ and $\phi_{x+y} h \neq 0$ then

$$
\Lambda_{i} \phi_{x+\delta_{i}+y} h=\phi_{y} \phi_{x} \Lambda_{i} \phi_{i} h=\phi_{y+x} h
$$

by the proof of Lemma 2 . So $\phi_{x+\delta_{i}+y} h \neq 0$.

Lemma 4. $1 \in \mathcal{H}_{e}$.

Proof. Let $h \in \mathcal{H}, h \leqslant 1$. Fix a state $i \in I$ and let $\tilde{h}_{i}: \mathbf{N}_{0}^{s} \rightarrow \mathbf{R}^{+}$be defined by $\tilde{h}_{i}(x)=h(x, i)$. Let $\tau_{0}=0$ and $\tau_{k}$ be the time of the $k$ th hitting of $i$ by the chain $Z_{n}, n \in \mathbf{N}$. It is well known that $X_{\tau_{k}}-X_{\tau_{k-1}}, k \in \mathbf{N}$ are independent identically distributed $\mathrm{N}_{0}^{s}$ valued random variables under the law $Q^{(y, i)}$ for every $y \in \mathbf{N}_{0}^{s}$. As $\left\{h\left(Y_{n}\right), n \in \mathbf{N}_{0}\right\}$ is a bounded martingale it follows that $\left\{\tilde{h}_{i}\left(X_{\tau_{k}}\right), k \in \mathbf{N}_{0}\right\}$ is a bounded martingale, so $\tilde{h_{i}}$ is bounded harmonic for the random walk $X_{\tau_{k}}, k \in \mathbf{N}_{0}$. Our assumptions on the $p_{i j}$ clearly imply that the support of the law of $X_{\tau_{1}}-X_{0}$ generates $Z^{s}$ as an Abelian group. So it follows from the Choquet-Deny theorem (see [7, p. 135]) that $\tilde{h}_{i}$ is a constant, say $c_{i}$. From harmonicity of $h$ it follows that $c_{i}=\sum_{j \in I} p_{i j} c_{j}$ for all $i \in I$, so all $c_{i}$ are the same. So $h$ is constant and it follows that $1 \in \mathcal{H}_{e}$.

Proof of TheOREM 1. The proof splits in two parts:

(I) For each $u \in\left(\mathbf{R}^{+}\right)^{s}-\{0\}, h_{u} \in \mathcal{H}_{e}$.

(II) If $h \in \mathcal{H}_{e}$ there is an $u \in\left(\mathbf{R}^{+}\right)^{s}-\{0\}$ with $h=h_{u}$.

Proof of (I). Let $u \in\left(\mathbf{R}^{+}\right)^{s}-\{0\}$ and $N_{u}=\left\{i \in I: u_{i}=0\right\}$. For $i, j \in N_{u}$ let $\tilde{p}_{i j}=p_{i j} u_{j} / v_{i} \neq 0$. The $\tilde{p}_{i j} ; i, j \in N_{u}^{c}$ form a Markov transition probability matrix on $N_{u}^{c}$.

Let $h \in \mathcal{H}, h \leqslant h_{u}$. Clearly $h(x, i)=0$ if $x_{j} \geqslant 1$ for some $j \in N_{u}$. It is then easy to see that $h / h_{u}$ restricted to elements $(x, i), i \notin N_{u}$ and $x_{j}=0$ for $j \in N_{u}$ is bounded harmonic for the space time process associated to the $\tilde{p}_{i j}$. From Lemma 4 (applied to $\tilde{p}$ ) there is a $c \in(0,1]$ with $h(x, i)=c h_{u}(x, i)$ for all $(x, i)$ satisfying the above restriction but clearly then for all $(x, i) \in \tilde{I}$ as for the other elements both sides are 0 . So $h_{u}$ is minimal.

Proof of (II). Let $h \in \mathcal{H}_{e}$. It is easy to see that $h(0, l) \neq 0$ for each $l \in I$. Indeed if $h(0, l)=0$ for some $l h$ would be identically 0 .

Let $N_{h}=\left\{i \in I: \phi_{i} h=0\right\}$ and $d=\sum_{i \in N_{h}^{c}} \delta_{i}$. From Lemma 3, $\phi_{d} h \neq 0$. Let $i \notin N_{k}$ and $i_{1}, \ldots, i_{k}$ be an enumeration of the elements of $N_{h}^{c}$ with $i_{k}=i$. Then for $x \in \mathbf{N}_{0}^{s}$,

$$
h(x, i) \geqslant p_{i i_{1}} p_{i_{1} i_{2}} \ldots p_{i_{k-1} i_{k}} h(x+d, i) .
$$

It follows that there is a $c>0$ such that $h(x, i) \geqslant \operatorname{ch}(x+d, i)$ for all $x$ and $i \notin N_{h}$. But then for all $(x, j) \in \tilde{I}$, 


$$
h(x, j)=\sum_{i \in N_{h}^{c}} p_{j i} h\left(x+\delta_{i}, i\right) \geqslant c \sum_{i \in N_{h}^{c}} p_{j i} h\left(x+d+\delta_{i}, i\right)=\operatorname{ch}(x+d, i)
$$

that is $h>c \phi_{d} h$. As $\phi_{d} h \neq 0$ there is a $c_{1}>0$ such that $h=c_{1} \phi_{d} h$ and then $h=c_{1}^{2} \phi_{d}^{2} h$.

Let now $l, i \notin N_{h}^{c}$ and let $i_{1}, \ldots, i_{2 k-1}$ be a sequence of elements in $N_{h}^{c}$ where each element of $N_{h}^{c}$ appears exactly twice except $i$ which appears once and further $i_{2 k-1}=l$. Then

$$
\left(\phi_{i} h\right)(x, l)=h\left(x+\delta_{i}, l\right) \geqslant p_{l i_{1}} p_{i_{1} i_{2}} \cdots p_{i_{2 k-2} i_{2 k-1}} h(x+2 d, l)
$$

for each $x \in \mathbf{N}_{0}^{s}$. So it follows as above that there is a $c_{2}>0$ with $\phi_{i} h>$ $c_{2} \phi_{d}^{2} h$. From $h=c_{1}^{2} \phi_{d}^{2} h$ it follows that $\phi_{d}^{2} h \neq 0$. So from Lemma 2 it follows that there is a constant $\lambda_{i}>0$ with $\phi_{i} h=\lambda_{i} h$. This obviously remains true for $i \in N_{h}$ and $\lambda_{i}=0$.

It follows that for $(x, l) \in \tilde{I}, h(x, l)=\lambda_{1}^{x_{1}} \lambda_{2}^{x_{2}} \cdots \lambda_{s}^{x_{s}} h(0, l)$. We further have the relation

$$
h(0, i)=\sum_{j \in I} p_{i j} h\left(\delta_{j}, j\right)=\sum_{j \in I} p_{i j} \lambda_{j} h(0, j)
$$

so putting $u_{i}=\lambda_{i} h(0, i), u=\left(u_{1}, \ldots, u_{s}\right)$ one easily obtains $h=h_{u}$.

Proof of Proposition 3. As $\tilde{\Delta}_{s}$ and $\Delta_{s}$ both are compact, it suffices to show that $\Psi$ is continuous and bijective. Clearly the $p_{i j}^{u}$ depend continuously on $u \in \tilde{\Delta}_{s}$ and the expectation of the recurrence times depend continuously on the transition probabilities. So the coefficients $\pi_{i}^{u}$ of the stationary distribution being the inverses of the expected recurrence times depend continuously on $u$. We now prove that $\Psi$ is onto. First, if $\pi \in \Delta_{s}$ has all components different from 0 it follows from Lemma 2.4 of [4] that there is a $u \in \tilde{\Delta}_{s}$ with $\pi=\pi^{u}$. If now $\pi$ has some components 0 , say $\pi_{1}=\cdots=\pi_{r}=$ $0(1<r<s)$. Then let $\left(\tilde{p}_{i j}\right)_{i, j=r+1, \ldots, s}$ be defined by

$$
\tilde{p}_{i j}=\frac{p_{i j}}{\sum_{k=r+1}^{s} p_{i k}}
$$

and let $u=\left(u_{r+1}, \ldots, u_{s}\right)$ with $\sum_{j=r+1}^{s} p_{1 j} u_{j}=1$ be such that $p_{i j}^{u}$ has stationary distribution $\left(\pi_{r+1}, \ldots, \pi_{s}\right)$ on $\{r+1, \ldots, s\}$. Then $u=$ $\left(0, \ldots, 0, u_{r+1}, \ldots, u_{s}\right)$ does it.

It remains to show that $\Psi$ is $1-1$. Let $u, u^{\prime} \in \Delta_{s}$ with $\pi^{u}=\pi^{u^{\prime}}$. We may assume that $u_{i} \neq 0$ for all $i \in I$. Now $p_{i j}^{u^{\prime}}=\left(p_{i j}^{u}\right)^{\left(u^{\prime} / u\right)}$ where $\left(u^{\prime} / u\right)_{i}=u_{i}^{\prime} / u_{i}$. So it follows from Lemmas 2.5 and 2.7 of [4] that

$$
\sum_{j} \log \left(\left(u_{j}^{\prime} / u_{j}\right)\left(\sum_{k} p_{j k}^{u}\left(u_{k}^{\prime} / u_{k}\right)\right)^{-1}\right) \pi_{j}^{u}=0
$$

or

$$
\sum_{j} \pi_{j}^{u} \log \left(u_{j}^{\prime} / u_{j}\right)=\sum_{j} \pi_{j}^{u} \log \left(\sum_{k} p_{j k}^{u}\left(u_{k}^{\prime} / u_{k}\right)\right) .
$$


Now if $u \neq u^{\prime}$ then $\log \left(\Sigma_{k} p_{j k}^{u}\left(u_{k}^{\prime} / u_{k}\right)\right)>\Sigma_{k} p_{j k}^{u} \log \left(u_{k}^{\prime} / u_{k}\right)$ so

$$
\begin{aligned}
\sum_{j} \pi_{j}^{u} \log \left(u_{j}^{\prime} / u_{j}\right) & >\sum_{j} \pi_{j}^{u} \sum_{k} p_{j k}^{u} \log \left(u_{k}^{\prime} / u_{k}\right) \\
& =\sum_{k} \pi_{k}^{u} \log \left(u_{k}^{\prime} / u_{k}\right)
\end{aligned}
$$

which is a contradiction.

\section{REFERENCES}

1. D. Blackwell and D. G. Kendall, The Martin boundary of Pólya's scheme and an application to stochastic population growth, J. Appl. Probability 1 (1964), 248-296.

2. G. Choquet, Lectures on analysis, Benjamin, Reading, Massachusetts, 1969.

3. J. N. Darroch and E. Seneta, On quasi-stationary distributions in absorbing discrete-time finite Markov chains, J. Appl. Probability 2 (1965), 88-100.

4. M. D. Donsker and S. R. S. Varadhan, Asymptotic evaluation of certain Markov process expectations for large time, Comm. Pure Appl. Math. 28 (1975), 1-47.

5. J. G. Kemeny, J. L. Snell and A. W. Knapp, Denumerable Markov chains, Springer, New York, 1976.

6. J. Lamperti and J. L. Snell, Martin boundaries for certain Markov chains, J. Math. Soc. Japan 15 (1963), 113-128.

7. D. Revuz, Markov chains, North-Holland, Amsterdam, 1975.

Fachgruppe Statistix, Universitït Konstanz, Postfach 7733 D-775 Konstanz, Germany 